\title{
Re-evaluation of Bleeding Events in the Japanese PRASFIT-Elective and PRASFIT-ACS Clinical Trials using the Bleeding Academic Research Consortium Criteria
}

Shunichi Miyazaki ${ }^{\star *}$, Takaaki Isshiki ${ }^{2}$, Takeshi Kimura ${ }^{3}$, Hisao Ogawa ${ }^{4}$, Hiroyoshi Yokoi ${ }^{5}$, Masakatsu Nishikawa ${ }^{6}$, Masato Nakamura ${ }^{7}$, Yuko Tanaka $^{8}$, and Shigeru Saito ${ }^{9}$, on behalf of the PRASFIT-ACS Investigators and PRASFIT-Elective Investigators

${ }^{1}$ Department of Medicine, Faculty of Medicine, Kinki University, Osaka, Japan

${ }^{2}$ Ageo Central General Hospital, Ageo, Japan

${ }^{3}$ Graduate School of Medicine, Kyoto University, Kyoto, Japan

${ }^{4}$ Department of Cardiovascular Medicine, Graduate School of Medical Sciences, Kumamoto University, Kumamoto and National Cerebral and Cardiovascular Center,

Suita, Osaka, Japan

${ }^{5}$ Fukuoka Sanno Hospital, Fukuoka, and International University of Health and Welfare, Tochigi, Japan

${ }^{6}$ Clinical Research Support Center, Mie University Hospital, Mie, Japan

${ }^{7}$ Division of Cardiovascular Medicine, Ohashi Medical Center, Toho University, Tokyo, Japan

${ }^{8}$ Clinical Data and Biostatistics Department, R\&D Division, Daiichi Sankyo Co., Ltd, Tokyo, Japan

${ }^{9}$ Division of Cardiology, Shonan Kamakura General Hospital, Kamakura, Japan

\begin{abstract}
Background: In 2011, the Bleeding Academic Research Consortium (BARC) criteria were published to standardize the assessment of bleeding events following PCI. However, the status of bleeding events as assessed using the BARC criteria is not established in Japan. The aim of this post-hoc analysis of the PRASFIT-ACS and PRASFIT-Elective trials was to re-classify the bleeding events from the Thrombolysis in Myocardial Infarction (TIMI) criteria into the BARC criteria.

Methods: Bleeding events had previously been assessed in both trials using the TIMI criteria. In the post-hoc analysis, the BARC criteria were applied retrospectively to each category of bleeding.

Results: In PRASFIT-ACS, the incidences of severe bleeds (combined type 3 or 5 bleeds according to BARC criteria) were 43/685 [6.3\%] with prasugrel and 37/678 [5.5\%] with clopidogrel [HR 1.071; 95\% Cl 0.668-1.667]. Types 3 or 5 events occurred at a higher rate closer to the time of PCI, and then plateaued. In PRASFIT-Elective, Type 3 bleeding occurred in 10/370 (2.7\%) patients in the prasugrel group and 12/372 (3.2\%) in the clopidogrel group. There was a higher incidence of bleeding events in PRASFIT-ACS than in PRASFIT-Elective, particularly more severe bleeds (combination of type 3 or 5 events).

Conclusions: The results obtained with the BARC criteria were similar to those reported using the original TIMI criteria. The incidences of type 3 or 5 events according to the BARC criteria were similar in the prasugrel and clopidogrel groups. Medical interventions might be needed during the acute period of $\mathrm{PCl}$ for ACS to reduce the risk of type 2 bleeding events in patients with low platelet aggregation.
\end{abstract}

Keywords: Bleeding events; Bleeding academic research consortium criteria; Clopidogrel; Prasugrel; Thrombolysis in myocardial infarction criteria

\section{Abbreviations}

PRASFIT-ACS: Prasugrel compared with clopidogrel For Japanese Patients with Acute Coronary Syndrome undergoing Percutaneous Coronary Intervention (PCI); PRASFIT-Elective: Prasugrel For Japanese patients with coronary artery diseases undergoing Elective PCI

\section{Introduction}

Percutaneous coronary intervention (PCI) is increasingly used to treat acute coronary syndrome (ACS) and stable angina in elective settings. However, major bleeding is a significant complication of PCI and antiplatelet therapy, and the mortality rate is consistently greater in patients with major or severe bleeding following PCI [1-3]. Patients undergoing PCI are usually started on dual antiplatelet therapy to prevent recurrent ischemia and stent thrombosis [4,5], with clopidogrel often used with aspirin. However, the responses to clopidogrel vary in Asian patients [6].

Prasugrel, a third-generation $\mathrm{P} 2 \mathrm{Y} 12$ receptor antagonist, has been associated with significantly fewer ischemic events but a higher incidence of bleeding than clopidogrel [7]. The most suitable dose of prasugrel for Japanese patients has been evaluated in two trials: the Efficacy and Safety of Adjusted-Dose Prasugrel Compared With Clopidogrel in Japanese Patients With Acute Coronary Syndrome (PRASFIT-ACS) [8] and Prasugrel in Patients With Coronary Artery Disease Undergoing Elective Percutaneous Coronary Intervention (PRASFIT-Elective) trials [9]. These trials found a low risk of clinically serious bleeding and confirmed the use of dose-adjusted prasugrel in

*Corresponding author: Dr. Shunichi Miyazaki, Division of Cardiology, Department of Medicine, Faculty of Medicine, Kinki University, 377-2 Ohnohigashi, Osakasayama, Osaka 589-8511, Japan, Tel: +81-72-366-0221; Fax: +81-72-3660206; E-mail: smiyazak@med.kindai.ac.jp

Received October 26, 2015; Accepted November 02, 2015; Published November 09, 2015

Citation: Miyazaki S, Isshiki T, Kimura T, Ogawa H, Yokoi H, et al. (2015) Reevaluation of Bleeding Events in the Japanese PRASFIT-Elective and PRASFITACS Clinical Trials using the Bleeding Academic Research Consortium Criteria. Cardiovasc Pharm Open Access 4: 162. doi:10.4172/2329-6607.1000162

Copyright: (c) 2015 Miyazaki S, et al. This is an open-access article distributed under the terms of the Creative Commons Attribution License, which permits unrestricted use, distribution, and reproduction in any medium, provided the original author and source are credited. 
Citation: Miyazaki S, Isshiki T, Kimura T, Ogawa H, Yokoi H, et al. (2015) Re-evaluation of Bleeding Events in the Japanese PRASFIT-Elective and PRASFIT-ACS Clinical Trials using the Bleeding Academic Research Consortium Criteria. Cardiovasc Pharm Open Access 4: 162. doi:10.4172/2329-6607.1000162

Page 2 of 8

patients undergoing elective PCI or those with ACS. Both PRASFITACS and PRASFIT-Elective used the Thrombolysis in Myocardial Infarction (TIMI) criteria to classify bleeding events, partly to ensure that the results could be compared with other leading international trials and because of the absence of other established criteria at their planning stages.

While the TIMI criteria are widely used in clinical trials $[2,7,10,11]$, they do have some limitations. In particular, bleeding events associated with a decrease in hemoglobin of $<3 \mathrm{mg} / \mathrm{dl}$ can be classified as "minor bleeding", but there are various types of minor bleeding in clinical settings, from severe to mild, so this definition may be too broad for the evaluation of the safety of drugs in clinical trials. Furthermore, the TIMI criteria were introduced in the fibrinolytic era, and may not reflect the types of bleeding events likely to occur during PCI and antiplatelet therapy [12]. Since the publication of the Bleeding Academic Research Consortium (BARC) criteria, several trials have evaluated the importance of post-PCI bleeding as a prognostic factor [13-16]. However, there are few comparisons of the incidences of bleeding events between the clinical conditions of ACS and following elective PCI with the same drug. Moreover, the present status of bleeding events as assessed using the BARC criteria in Japan is not established. Therefore, we conducted a post-hoc analysis of the PRASFIT-ACS and PRASFITElective trials to re-evaluate bleeding events using the BARC criteria. A combined analysis was not feasible because of slight differences in the designs of each study and was not deemed appropriate because it may mask differences in bleeding complications between PCI performed in elective and ACS settings.

\section{Methods}

\section{Design of PRASFIT-ACS and PRASFIT-Elective}

PRASFIT-ACS [8] and PRASFIT-Elective [9] were both multicenter, randomized, double-blind, parallel-group trials. The primary efficacy endpoint in both trials was the incidence of major adverse cardiovascular events, defined as a composite of cardiovascular death, nonfatal myocardial infarction, and nonfatal ischemic stroke. The safety endpoint was non-coronary artery bypass graft (CABG)related bleeding events. Both trials were approved by ethics committees at each participating institution and were conducted in accordance with national and international regulations. Full details of the trials are reported in more detail elsewhere [8,9], and an overview of each study design is provided here.

PRASFIT-ACS was conducted at 162 centers throughout Japan between June 2010 and December 2012. Participants were Japanese ACS patients scheduled for coronary artery stenting. Patients received a loading dose of prasugrel $20 \mathrm{mg}$ or clopidogrel $300 \mathrm{mg}$ before PCI, except in urgent cases where it was administered as soon as possible afterwards. Clinical trial registration: JapicCTI-101339 (Japan Pharmaceutical Information Center).

PRASFIT-Elective was conducted at 100 centers throughout Japan between August 2011 and December 2012. The participants were Japanese stable CAD patients scheduled for elective PCI. Patients were randomized to receive either prasugrel or clopidogrel. The allocated loading dose of the study drug (20 mg prasugrel or $300 \mathrm{mg}$ clopidogrel) was given before PCI. Unlike PRASFIT-ACS, patients could be started on the maintenance dose without the loading dose, in which case the maintenance dose was administered for 14-21 days before PCI. Clinical trial registration: JapicCTI-111550 (Japan Pharmaceutical Information Center).
Both trials consisted of a 24- to 48-week treatment period followed by a 2-week untreated observation period. A maintenance dose of prasugrel $(3.75 \mathrm{mg})$ or clopidogrel $(75 \mathrm{mg})$ was administered once daily after breakfast for 24-48 weeks, starting on the day after the loading dose, and was continued for the remainder of the treatment period. Aspirin (81-330 mg for the first dose and $81-100 \mathrm{mg} /$ day thereafter) was concomitantly administered during the treatment period in both trials.

\section{Bleeding criteria}

In both PRASFIT-ACS and PRASFIT-Elective, the safety endpoint was the incidence of non-CABG-related bleeding events occurring up until 2 weeks after the last dose. Five categories of bleeding events were evaluated:

1. non-CABG-related Thrombolysis in Myocardial Infarction (TIMI) major bleeding (major bleeding): intracranial or clinically significant bleeding with a decrease in hemoglobin $\geq 5 \mathrm{~g} / \mathrm{dl}$;

2. non-CABG-related TIMI minor bleeding (minor bleeding): clinically significant bleeding with a decrease in hemoglobin $3-<5 \mathrm{~g} / \mathrm{dl}$;

3. clinically relevant non-major or minor bleeding: bleeding from critical sites (e.g., retroperitoneal, intrapericardial, intravitreal/ retinal, intraspinal, and intra-articular hemorrhage); gastrointestinal bleeding accompanied by decreased hemoglobin; gross hematuria not attributed to external factors (e.g., urinary catheterization); epistaxis requiring otolaryngology; gingival bleeding requiring dental treatment; and bleeding requiring discontinuation of the study treatment at the investigator's discretion; these bleeding events were accompanied by a decrease in hemoglobin $<3 \mathrm{~g} / \mathrm{dl}$;

4. other bleeding: bleeding events not satisfying criteria 1-3;

5. life-threatening bleeding: a composite of fatal bleeding, bleeding requiring intravenous inotropic medication, and bleeding requiring the transfusion of $\geq 4$ units of red blood cells.

In the current post-hoc analysis, we sought to re-evaluate the bleeding events using the BARC criteria (Table 1). We assessed each bleeding event and re-classified them as types 2 to 5 according to the BARC criteria, or as types 3 or 5 combined. Type 1 events do not require treatment and were not assessed in this analysis. The rationale for combining types 3 or 5 was to evaluate the most severe bleeds together, and to determine whether severe bleeding events constituted spontaneous bleeding or were a complication of the PCI procedure itself (or a result of other external factors, such as trauma or surgery). Bleeding events were assessed by a Safety Event Committee using blinded data.

\section{Statistical analysis}

All analyses were performed using the safety analysis set (i.e. patients who took the study drug at least once). Descriptive statistics were used to summarize baseline characteristics and are presented as the $\mathrm{n}(\%)$ and/or mean \pm standard deviation. The incidence of bleeding events was calculated for each group. Hazard ratios (HRs) with twosided $95 \%$ confidence intervals (CIs) for the prasugrel group relative to the clopidogrel group were calculated for bleeding events using the Cox proportional hazard model with adjustment for weight ( $>50$ vs. $\leq 50 \mathrm{~kg}$ ) and puncture site (femoral artery, yes vs. no; including no first revascularization) and the generalized Wilcoxon test was used to compare the two groups in PRASFIT-ACS. Bleeding events were also assessed according to external factors (e.g., as a complication of PCI). The cumulative incidence of type 3 or type 5 bleeding events according 
Citation: Miyazaki S, Isshiki T, Kimura T, Ogawa H, Yokoi H, et al. (2015) Re-evaluation of Bleeding Events in the Japanese PRASFIT-Elective and PRASFIT-ACS Clinical Trials using the Bleeding Academic Research Consortium Criteria. Cardiovasc Pharm Open Access 4: 162. doi:10.4172/2329-6607.1000162

Page 3 of 8

to the BARC criteria was plotted for both treatment groups in each study using the Kaplan-Meier method. Patients with a primary event were censored from the analysis at the time of the event. Intergroup comparisons were performed only in PRASFIT-ACS. In PRASFITElective, clopidogrel was only used as a reference drug because it was not indicated for elective PCI at the time of the study; therefore, statistical tests were not conducted for comparisons between prasugrel and clopidogrel. Receiver operator characteristic (ROC) curve analysis was conducted to determine the cutoff values of platelet reactivity units (PRU; determined by VerifyNow assays) for the incidence of type 2 bleeding events. Two ROC curves were plotted: (1) PRU at 5-12 h after the $\mathrm{LD}$ and the incidence of type 2 bleeding events within 3 days after PCI (acute antiplatelet therapy); and (2) PRU at 4 weeks after PCI and the incidence of type 2 bleeding events between 4 days after PCI and the end of treatment (steady state antiplatelet therapy). All analyses were performed used SAS version 9.2 (SAS Institute, Cary, NC, USA).

\section{Results}

\section{Patient characteristics}

Table 2 shows the characteristics of the evaluable study populations from PRASFIT-ACS and PRASFIT-Elective.

A total of 1,363 patients in PRASFIT-ACS received one dose of either prasugrel $(n=685)$ or clopidogrel $(n=678)$ and were included in the safety evaluation. There were 183 and 190 patients in the prasugrel and clopidogrel groups, respectively, who discontinued therapy after starting the study drug. The mean duration of study drug administration was 213.5 days in the prasugrel group and 207.5 days in the clopidogrel group. The baseline demographics were similar in both treatment groups. The mean age was 65 years, the mean body weight was $64 \mathrm{~kg}$, and $21 \%$ of patients were female. About half of the patients in each treatment group had ST-segment elevation myocardial infarction and half had unstable angina and/or non-ST-segment elevation myocardial infarction.

In PRASFIT-Elective, 742 patients were included in the efficacy and safety evaluations (prasugrel, $n=370$; clopidogrel, $n=372$ ). The mean duration of exposure was similar in both groups. All patients were followed up for at least 24 weeks, with 179 being followed up for 24-48 weeks. There were no marked differences in the main characteristics of the two groups. Mean age and weight were 67 years and $64 \mathrm{~kg}$, respectively, and $30 \%$ of the patients were women. Approximately $80 \%$ of patients had stable angina. The primary revascularization procedure was similar between the two groups, with drug-eluting stents used in $\geq 90 \%$ of patients in each group.

\section{Incidence of bleeding events defined using the original criteria}

Table 3 shows the incidences of bleeding events in PRASFIT-ACS and PRASFIT-Elective using the original study criteria. There were four fatal bleeding events in PRASFIT-ACS and none in PRASFITElective corresponding to BARC type $5 \mathrm{a}$.

In PRASFIT-ACS, TIMI major bleeding events occurred in 13 (1.9\%) patients in the prasugrel group and $15(2.2 \%)$ patients in the clopidogrel group (HR 0.82; 95\% CI 0.39-1.73). Spontaneous TIMI major bleeding occurred in $4(0.6 \%)$ patients in the prasugrel group

\begin{tabular}{|c|c|}
\hline Type & Definition \\
\hline Type 0 & No bleeding \\
\hline Type 1 & $\begin{array}{l}\text { Bleeding that is not actionable and does not cause the patient to seek unscheduled performance of studies, hospitalization, or treatment } \\
\text { by a healthcare professional; may include episodes leading to self-discontinuation of medical therapy by the patient without consulting a } \\
\text { healthcare professional }\end{array}$ \\
\hline Type 2 & $\begin{array}{l}\text { Any overt, actionable sign of hemorrhage (e.g., more bleeding than would be expected for a clinical circumstance, including bleeding } \\
\text { found by imaging alone) that does not fit the criteria for type } 3,4 \text {, or } 5 \text { but does meet at least one of the following criteria: (1) requiring } \\
\text { nonsurgical, medical intervention by a healthcare professional, (2) leading to hospitalization or increased level of care, or ( } 3 \text { ) prompting } \\
\text { evaluation }\end{array}$ \\
\hline \multicolumn{2}{|l|}{ Type 3} \\
\hline Type $3 a$ & $\begin{array}{l}\text { Overt bleeding plus hemoglobin drop of } 3 \text { to }<5 \mathrm{~g} / \mathrm{dL}^{*} \text { (provided hemoglobin drop is related to bleed) } \\
\text { Any transfusion with overt bleeding }\end{array}$ \\
\hline Type 3b & $\begin{array}{l}\text { Overt bleeding plus hemoglobin drop } \geq 5 \mathrm{~g} / \mathrm{dL}^{*} \text { (provided hemoglobin drop is related to bleed) } \\
\text { Cardiac tamponade } \\
\text { Bleeding requiring surgical intervention for control (excluding dental/nasal/skin/hemorrhoid) } \\
\text { Bleeding requiring intravenous vasoactive agents }\end{array}$ \\
\hline Type 3c & $\begin{array}{l}\text { Intracranial hemorrhage (does not include microbleeds or hemorrhagic transformation, does include intraspinal) } \\
\text { Subcategories confirmed by autopsy or imaging or lumbar puncture } \\
\text { Intraocular bleed compromising vision }\end{array}$ \\
\hline Type 4: CABG-related bleeding & $\begin{array}{l}\text { Perioperative intracranial bleeding within } 48 \mathrm{~h} \\
\text { Reoperation after closure of sternotomy for the purpose of controlling bleeding } \\
\text { Transfusion of } \geq 5 \mathrm{U} \text { whole blood or packed red blood cells within a } 48 \mathrm{~h} \text { period } \\
\text { Chest tube output } \geq 2 \mathrm{~L} \text { within a } 24 \mathrm{~h} \text { period }\end{array}$ \\
\hline \multicolumn{2}{|l|}{ Type 5: Fatal bleeding } \\
\hline Type 5a & Probable fatal bleeding; no autopsy or imaging confirmation but clinically suspicious \\
\hline Type 5b & Definite fatal bleeding; overt bleeding or autopsy or imaging confirmation \\
\hline
\end{tabular}

Platelet transfusions should be recorded and reported but are not included in these definitions until further information is obtained about the relationship to outcomes. If a CABG-related bleed is not adjudicated as at least a type 3 severity event, it will be classified as not a bleeding event. If a bleeding event occurs with a clear temporal relationship to CABG (i.e., within a 48-h time frame) but does not meet type 4 severity criteria, it will be classified as not a bleeding event.

${ }^{*}$ Corrected for transfusion ( $1 \mathrm{U}$ packed red blood cells or $1 \mathrm{U}$ whole blood $=1 \mathrm{~g} / \mathrm{dL}$ hemoglobin).

†Cell saver products are not counted.

CABG = coronary artery bypass graft

Reproduced with permission from Mehran et al (reference \#12). Standardized bleeding definitions for cardiovascular clinical trials: a consensus report from the Bleeding Academic Research Consortium, Circulation 123: 2736-2747. Promotional and commercial use of the material in print, digital or mobile device format is prohibited without permission from Wolters Kluwer Health. Please contact Iwwjournalpermissions@wolterskluwer.com for further information.

Table 1: Bleeding Academic Research Consortium criteria. 
Citation: Miyazaki S, Isshiki T, Kimura T, Ogawa H, Yokoi H, et al. (2015) Re-evaluation of Bleeding Events in the Japanese PRASFIT-Elective and PRASFIT-ACS Clinical Trials using the Bleeding Academic Research Consortium Criteria. Cardiovasc Pharm Open Access 4: 162. doi:10.4172/2329-6607.1000162

Page 4 of 8

\begin{tabular}{|c|c|c|c|c|}
\hline & \multicolumn{2}{|c|}{ PRASFIT-ACS } & \multicolumn{2}{|c|}{ PRASFIT-Elective } \\
\hline & $\begin{array}{c}\text { Prasugrel } \\
(n=685)\end{array}$ & $\begin{array}{c}\text { Clopidogrel } \\
(n=678)\end{array}$ & $\begin{array}{c}\text { Prasugrel } \\
(n=370)\end{array}$ & $\begin{array}{c}\text { Clopidogrel } \\
(n=372)\end{array}$ \\
\hline Female & $149(21.8)$ & $140(20.6)$ & $96(25.9)$ & $109(29.3)$ \\
\hline \multicolumn{5}{|l|}{ Age, years } \\
\hline Mean \pm SD & $65.4 \pm 11.4$ & $65.1 \pm 11.3$ & $67.5 \pm 7.5$ & $67.4 \pm 7.4$ \\
\hline$\geq 75$ years & $165(24.1)$ & $148(21.8)$ & $86(23.2)$ & $93(25.0)$ \\
\hline Body weight (kg) & $64.2 \pm 12.3$ & $64.4 \pm 11.2$ & $64.0 \pm 10.9$ & $63.7 \pm 11.5$ \\
\hline$\leq 50$ & $85(12.4)$ & $72(10.6)$ & $34(9.2)$ & $40(10.8)$ \\
\hline$\leq 60$ & $266(38.9)$ & $240(35.4)$ & $131(35.4)$ & $146(39.2)$ \\
\hline Body mass index $\left(\mathrm{kg} / \mathrm{m}^{2}\right)$ & $24.2 \pm 3.6$ & $24.2 \pm 3.3$ & $24.49 \pm 3.14$ & $24.64 \pm 3.42$ \\
\hline Current smoker & $273(39.9)$ & $279(41.2)$ & $67(18.1)$ & $63(16.9)$ \\
\hline \multicolumn{5}{|l|}{ ACS type } \\
\hline UA/NSTEMI & $343(50.1)$ & $337(49.7)$ & - & - \\
\hline UA & $156(22.8)$ & $124(18.3)$ & - & - \\
\hline NSTEMI & $187(27.3)$ & $213(31.4)$ & - & - \\
\hline STEMI & $340(49.6)$ & $341(50.3)$ & - & - \\
\hline \multicolumn{5}{|l|}{ Underlying disease } \\
\hline Stable angina & - & - & 277 (74.9) & $284(76.3)$ \\
\hline Prior Ml & - & - & $21(5.7)$ & $16(4.3)$ \\
\hline UA & - & - & $30(8.1)$ & $35(9.4)$ \\
\hline Silent myocardial ischemia & - & - & $41(11.1)$ & $33(8.9)$ \\
\hline \multicolumn{5}{|l|}{ No. of treated lesion sites (first $\mathrm{PCl}$ ) } \\
\hline 1 & $470(68.6)$ & $455(67.1)$ & $225(60.8)$ & $210(56.5)$ \\
\hline$>1$ & $181(26.4)$ & $182(26.8)$ & $136(36.8)$ & $139(37.4)$ \\
\hline No first $\mathrm{PCl}$ & $34(5.0)$ & $41(6.0)$ & $9(2.4)$ & $139(37.4)$ \\
\hline \multicolumn{5}{|l|}{ Stent type (first PCl) } \\
\hline Bare metal stent & $353(55.2)$ & $353(56.3)$ & $37(10.3)$ & $28(8.2)$ \\
\hline Drug-eluting stent & $291(45.5)$ & $278(44.3)$ & $324(90.5)$ & $319(93.0)$ \\
\hline \multicolumn{5}{|l|}{ Arterial access route } \\
\hline Femoral & $366(53.4)$ & $353(52.1)$ & $75(20.3)$ & $65(17.5)$ \\
\hline Radial & $285(41.6)$ & $279(41.2)$ & $258(69.7)$ & $258(69.4)$ \\
\hline Hypertension & $495(72.3)$ & $491(72.4)$ & $295(79.7)$ & $304(81.7)$ \\
\hline Dyslipidemia & $516(75.3)$ & $500(73.7)$ & $296(80.0)$ & $305(82.0)$ \\
\hline Diabetes mellitus & $250(36.5)$ & $237(35.0)$ & $150(40.5)$ & $132(35.5)$ \\
\hline Prior MI & $34(5.0)$ & $35(5.2)$ & $88(23.8)$ & $66(17.6)$ \\
\hline Prior ischemic stroke & $1(0.1)$ & $7(1.0)$ & $14(3.8)$ & $9(2.4)$ \\
\hline Prior transient ischemic attack & - & - & $3(0.8)$ & $2(0.5)$ \\
\hline Asymptomatic cerebral infarction & $25(3.6)$ & $24(3.5)$ & $20(5.4)$ & $29(7.8)$ \\
\hline Prior revascularizations & $40(5.8)$ & $45(6.6)$ & $124(33.5)$ & $123(33.1)$ \\
\hline Prior CABG & $6(0.9)$ & $4(0.6)$ & $8(2.2)$ & $2(0.5)$ \\
\hline Prior TLR & $15(2.2)$ & $13(1.9)$ & $39(7.8)$ & $37(9.9)$ \\
\hline $\mathrm{CrCl}(\mathrm{ml} / \mathrm{min})$ & $n=622$ & $\mathrm{n}=625$ & $n=300$ & $\mathrm{n}=307$ \\
\hline$>60$ & $486(78.1)$ & $507(81.1)$ & $236(78.7)$ & $235(76.5)$ \\
\hline$\leq 60$ & $136(21.9)$ & $118(18.9)$ & $64(21.3)$ & $72(23.5)$ \\
\hline \multicolumn{5}{|l|}{ Concomitant therapy } \\
\hline Proton pump inhibitor & $282(41.2)$ & $287(42.3)$ & $181(48.9)$ & $185(49.7)$ \\
\hline Statin & $346(50.5)$ & $328(48.4)$ & $248(67.0)$ & $254(68.3)$ \\
\hline Calcium channel blocker & $184(26.9)$ & $159(23.5)$ & $178(48.1)$ & $176(47.3)$ \\
\hline$\beta$-blocker & $122(17.8)$ & $114(16.8)$ & $132(35.7)$ & $268(72.0)$ \\
\hline \multicolumn{5}{|l|}{ Revascularization vessel } \\
\hline Right coronary artery & $216(31.5)$ & $222(32.7)$ & $120(32.4)$ & $109(29.3)$ \\
\hline Left main coronary trunk & $13(1.9)$ & $10(1.5)$ & $1(0.3)$ & $1(0.3)$ \\
\hline LAD & $343(50.1)$ & $323(47.6)$ & $179(48.4)$ & $182(48.9)$ \\
\hline Left circumflex coronary artery & $107(15.6)$ & $107(15.8)$ & $90(24.3)$ & $83(22.3)$ \\
\hline
\end{tabular}

Values are $\mathrm{n}(\%)$ or mean \pm standard deviation, or median (min-max). Differences between the two treatment groups in PRASFIT-Elective were not tested for statistical significance.

$\mathrm{ACS}=$ acute coronary syndrome; $\mathrm{CABG}=$ coronary artery bypass grafting; $\mathrm{CrCl}=$ creatinine clearance (estimated using the Cockcroft-Gault formula); $\mathrm{LAD}=$ left anterior descending coronary artery; $\mathrm{MI}=$ myocardial infarction; NSTEMI = non-ST-segment elevation myocardial infarction; $\mathrm{PCI}=$ percutaneous coronary intervention; STEMI = ST-segment elevation myocardial infarction; TLR = target lesion revascularization; UA = unstable angina.

Reproduced with permission from Saito et al (reference \#8), Efficacy and safety of adjusted-dose prasugrel compared with clopidogrel in Japanese patients with acute coronary syndrome, Circ J 78:1684-92.

Table 2: Baseline characteristics of patients in PRASFIT-ACS and PRASFIT-Elective.

(Safety Analysis Set) 
Citation: Miyazaki S, Isshiki T, Kimura T, Ogawa H, Yokoi H, et al. (2015) Re-evaluation of Bleeding Events in the Japanese PRASFIT-Elective and PRASFIT-ACS Clinical Trials using the Bleeding Academic Research Consortium Criteria. Cardiovasc Pharm Open Access 4: 162. doi:10.4172/2329-6607.1000162

Page 5 of 8

\begin{tabular}{|c|c|c|c|c|c|}
\hline \multirow[t]{2}{*}{ Bleeding event } & \multicolumn{3}{|c|}{ PRASFIT-ACS } & \multicolumn{2}{|c|}{ PRASFIT-Elective } \\
\hline & $\begin{array}{c}\text { Prasugrel } \\
(n=685)\end{array}$ & $\begin{array}{c}\text { Clopidogrel } \\
(n=678)\end{array}$ & $\mathrm{HR}(95 \% \mathrm{Cl})$ & $\begin{array}{l}\text { Prasugrel } \\
(n=370)\end{array}$ & $\begin{array}{c}\text { Clopidogrel } \\
(n=372)\end{array}$ \\
\hline TIMI major bleeding & $13(1.9)$ & $15(2.2)$ & $0.82(0.39-1.73)$ & $0(0.0)$ & $8(2.2)$ \\
\hline Life-threatening bleeding & $4(0.6)$ & $7(1.0)$ & $0.54(0.16-1.85)$ & - & - \\
\hline Fatal bleeding & $2(0.3)$ & $1(0.1)$ & $1.77(0.16-19.54)$ & - & - \\
\hline TIMI minor bleeding & $27(3.9)$ & $15(2.2)$ & $1.76(0.94-3.31)$ & $6(1.6)$ & $3(0.8)$ \\
\hline Clinically relevant bleeding & $29(4.2)$ & $39(5.8)$ & $0.72(0.44-1.16)$ & $14(3.8)$ & $12(3.2)$ \\
\hline Other bleeding & $298(43.5)$ & $209(30.8)$ & $1.51(1.26-1.80)$ & $130(35.1)$ & $118(31.7)$ \\
\hline Overall bleeding events & $341(49.8)$ & $247(36.4)$ & $1.48(1.25-1.74)$ & $141(38.1)$ & $128(34.4)$ \\
\hline Bleeding events leading to discontinuation & $16(2.3)$ & $20(2.9)$ & $0.76(0.40-1.48)$ & $9(2.4)$ & $9(2.4)$ \\
\hline TIMI major or minor bleeding & $39(5.7)$ & $29(4.3)$ & $1.30(0.81-2.11)$ & $6(1.6)$ & $11(3.0)$ \\
\hline Major, minor or clinically relevant bleeding & $66(9.6)$ & $65(9.6)$ & $0.98(0.70-1.38)$ & $20(5.4)$ & $23(6.2)$ \\
\hline
\end{tabular}

${ }^{*}$ Incidence $(\%)=\mathrm{n}$ for each bleeding event/n for each group (prasugrel or clopidogrel) $\times 100 \%$.

In PRASFIT-Elective, clinically relevant bleeding included: bleeding at important sites (e.g., retroperitoneum, in the pericardial cavity, vitreous body, retina, intraspinal cavity, intra-articular); gastrointestinal bleeding with decreased hemoglobin; macroscopic hematuria not attributable to external factor; epistaxis requiring otorhinolaryngeal treatment; gingival bleeding requiring dental treatment; bleeding considered necessary to discontinue or interrupt administration by investigator or subinvestigator.

$\mathrm{TIMI}=$ Thrombolysis in Myocardial Infarction; HR = hazard ratio.

Reproduced with permission from Saito et al (reference \#8), Efficacy and safety of adjusted-dose prasugrel compared with clopidogrel in Japanese patients with acute coronary syndrome, Circ J 78:1684-92.

Table 3: Incidence of bleeding events defined using the original criteria in PRASFIT-ACS and PRASFIT-Elective.

and $6(0.9 \%)$ patients in the clopidogrel group (HR 0.64; 95\% CI $0.18-$ 2.27). Any TIMI minor bleeding occurred in 27 (3.9\%) patients in the prasugrel group and 15 (2.2) patients in the clopidogrel group (HR 1.76; 95\% CI 0.94-3.31). Spontaneous TIMI minor bleeding occurred in $7(1.0 \%)$ patients in the prasugrel group and $6(0.9 \%)$ patients in the clopidogrel group (HR 1.10; 95\% CI 0.37-3.27). The number of patients with any bleeding was $341(49.8 \%)$ in the prasugrel group and 247 (36.4) in the clopidogrel group (HR 1.48; 95\% CI 1.25-1.74). The number of patients with spontaneous bleeding was $115(16.8 \%)$ in the prasugrel group and $103(15.2 \%)$ in the clopidogrel group (HR 1.09; 95\% CI 0.83-1.42).

In PRASFIT-Elective, TIMI major bleeding occurred in $0(0 \%)$ patients in the prasugrel group and in $8(2.2 \%)$ patients in the clopidogrel group. In the prasugrel and clopidogrel groups, the incidence rates of TIMI minor bleeding were $1.6 \%$ and $0.8 \%$, respectively, and those for all bleeding events were $38.1 \%$ and $34.4 \%$, respectively. Bleeding events as a complication of PCI (TIMI major or minor) occurred in $0.8 \%$ of patients in the prasugrel group and in $0.5 \%$ of patients in the clopidogrel group. Very few bleeding events were classified as spontaneous or with an exogenous cause.

\section{Incidence of bleeding events defined using the BARC criteria}

We then reclassified the bleeding events using the BARC criteria. Figure 1 shows how the BARC criteria were applied to the original results relating to number of bleeds from PRASFIT-ACS and PRASFITElective. Table 4 summarizes the incidence of bleeding using the BARC criteria in PRASFIT-ACS and PRASFIT-Elective.

In PRASFIT-ACS, type 5 BARC bleeding occurred in 6 patients overall: $4 / 685(0.6 \%)$ in the prasugrel group (3 with cardiac rupture and 1 with brain stem hemorrhage) and 2/678 (0.3\%) in the clopidogrel group (1 each with cardiac rupture and left ventricular inferior wall rupture). Type 4 bleeding (bleeding associated with CABG) occurred in a total of 11 patients: 7/685 (1.0\%) in the prasugrel group and 4/678 $(0.6 \%)$ in the clopidogrel group. Type 3 bleeding occurred in $39 / 685$ (5.7\%) patients in the prasugrel group and 35/678 (5.2\%) in the clopidogrel group. Severe bleeding, which was defined as the total of BARC type 3 or 5 bleeds, occurred in 43/685 (6.3\%) patients in the prasugrel group and $37 / 678$ (5.5\%) patients in the clopidogrel group (HR 1.071; 95\% CI 0.668-1.667). The Kaplan-Meier curve used to determine the time-point of the bleeding event relative to PCI is shown in Figure 2. Type 3 or 5 events occurred at a higher rate closer to the time of PCI, and then plateaued (Figure 2a). Type 2 BARC bleeding occurred in $73 / 685$ (10.7\%) prasugrel patients and in 44/678 (6.5\%) clopidogrel patients (HR 1.654; 95\% CI 1.138-2.404).

In PRASFIT-Elective, there were no instances of type 4 or 5 bleeding. Type 3 bleeding occurred in 10/370 (2.7\%) patients in the prasugrel group and in $12 / 372(3.2 \%)$ patients in the clopidogrel group. Type 3 bleeding (there were no type 5 events) occurred at a gradual rate throughout the study (Figure 2b). Type 2 bleeding occurred in 31/370 (8.4\%) patients in the prasugrel group and $23 / 372(6.2 \%)$ patients in the clopidogrel group.

The incidences of types 3,4 , and 5 events were not significantly different between the prasugrel and clopidogrel groups in PRASFITACS, and were numerically similar between the two groups in PRASFIT-Elective. The number of type 2 events in PRASFIT-ACS was significantly greater in the prasugrel group than in the clopidogrel group.

\section{ROC curve analysis of PRU and Type 2 bleeding events}

To examine whether the extent of platelet inhibition is possibly associated with a higher incidence of type 2 bleeding events, we performed ROC curve analysis for bleeding events in the acute and steady state periods of antiplatelet therapy. The ROC curve analysis for acute antiplatelet therapy (PRU at 5-12 $\mathrm{h}$ and bleeding events within 3 days after the LD; Figure 3a) yielded a cutoff value of PRU was 210 (area under the curve: 0.6425 ). The incidence of type 2 bleeding events was $5.6 \%$ and $1.3 \%$ for PRU $\leq 210$ and $>210$, respectively $(P=0.0017$; generalized Wilcoxon test). Meanwhile, the ROC curve analysis for steady state antiplatelet therapy (PRU at 4 weeks and bleeding events from 4 days to the end of treatment; Figure $3 \mathrm{~b}$ ) yielded a cutoff value of 139 (area under the curve: 0.5424 ). The incidences of type 2 bleeding events were $9.6 \%$ and $5.7 \%$ for PRU $\leq 139$ and $>139$, respectively $(P=0.0825$; generalized Wilcoxon test $)$.

\section{Bleeding events in relation to external factors}

Bleeding events were also evaluated in relation to the presence or absence of external factors (Supplementary Table 1). In the absence of external factors, the incidence of bleeding events was similar between 
Citation: Miyazaki S, Isshiki T, Kimura T, Ogawa H, Yokoi H, et al. (2015) Re-evaluation of Bleeding Events in the Japanese PRASFIT-Elective and PRASFIT-ACS Clinical Trials using the Bleeding Academic Research Consortium Criteria. Cardiovasc Pharm Open Access 4: 162. doi:10.4172/2329-6607.1000162

Page 6 of 8

the prasugrel and clopidogrel groups. However, in the presence of external factors, the incidence of type 2 bleeds in PRASFIT-ACS was significantly higher in the prasugrel group (60/685 [8.8\%]) than in the clopidogrel group $(26 / 678[3.8 \%])$. The bleeding events that occurred in the presence of external factors were then analyzed by the type of external factor (PCI complication or other). Many of the type 2 bleeding events in the prasugrel group were found to be associated with PCI technique.

\section{Discussion}

This post-hoc analysis was conducted to assess the incidence of bleeding in PRASFIT-ACS and PRASFIT-Elective using the BARC criteria. The aim was to determine whether these new, standardized criteria could be applied successfully to Japanese patients with ACS or undergoing elective procedures. Severe bleeding following PCI is

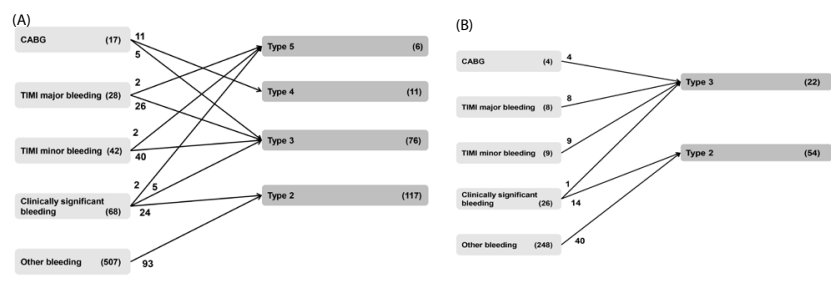

Figure 1: Reclassification of bleeding events using the Bleeding Academic Research Consortium criteria. (a) PRASFIT-ACS. (b) PRASFIT-Elective.

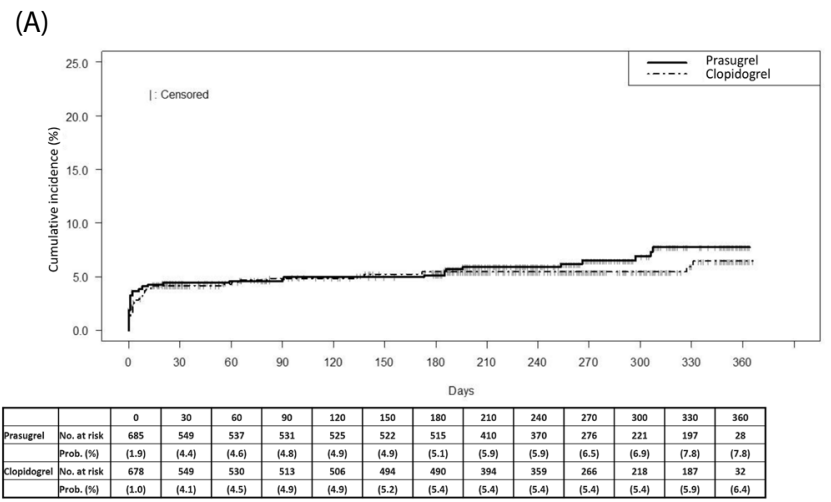

(B)

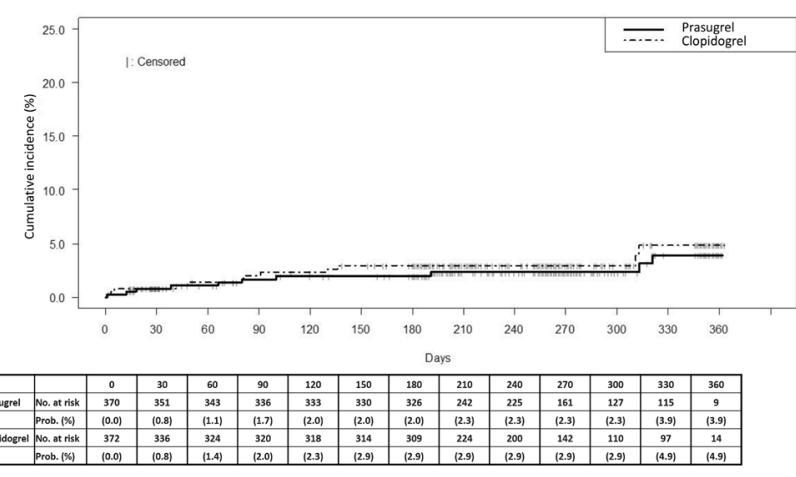

Figure 2: Cumulative incidence of type 3 or 5 bleeding events (Bleeding Academic Research Consortium Criteria). (a) PRASFIT-ACS. (b) PRASFIT-Elective

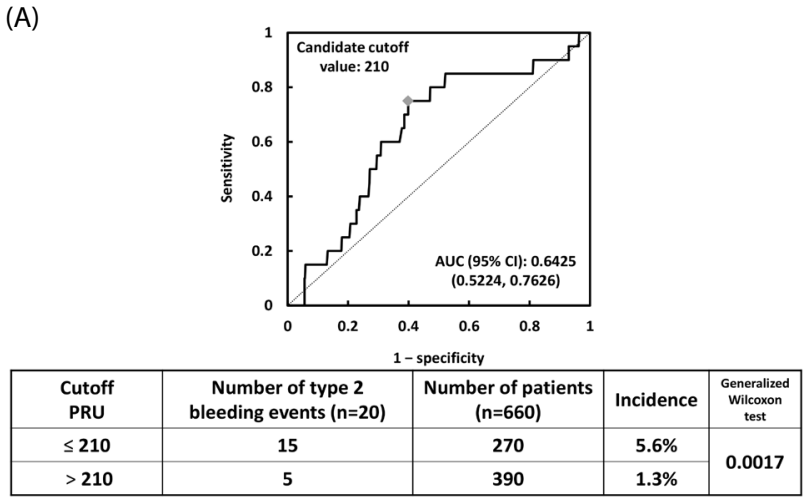

(B)

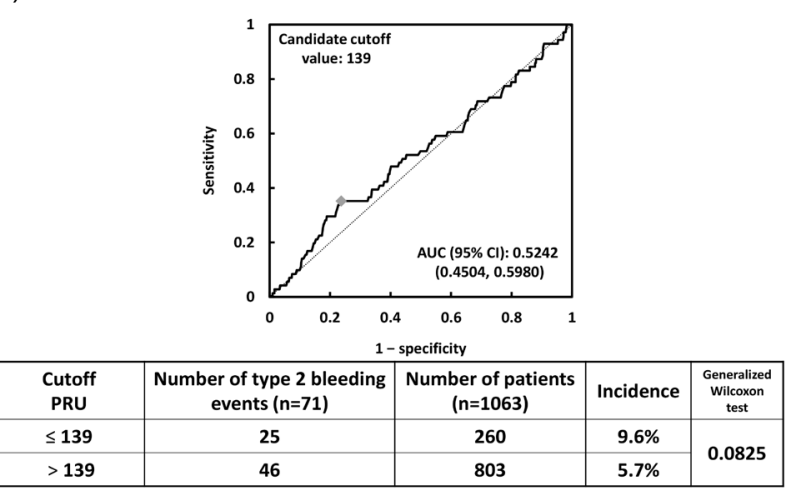

Figure 3: Receiver operator characteristic curve analysis of type 2 bleeding events (Bleeding Academic Research Consortium criteria). (a) Acute antiplatelet therapy (platelet reactivity units at 5-12 $\mathrm{h}$ and bleeding events within 4 days after the loading dose). (b) Steady state antiplatelet therapy (platelet reactivity units at 4 weeks and bleeding events from 4 days to the end of treatment).

a significant predictor of adverse outcomes following PCI including myocardial infarction, stroke, stent thrombosis and death [1-3]. Recent trials using the BARC criteria have found that the more serious the bleed, the higher the risk of mortality $[14,15]$.

In both PRASFIT-ACS and in PRASFIT-Elective, the incidence of type 3 BARC bleeds was similar after treatment with clopidogrel and prasugrel. Type 5 BARC bleeds (fatal bleeds) also occurred at a similar rate in both treatment groups in PRASFIT-ACS. These rates of serious bleeds were similar to that found with the original TIMI criteria in these trials. Type 2 bleeding occurred to a greater extent in the prasugrel groups in PRASFIT-ACS. The BARC type 2 bleeds are those considered related to the effects of drug used during and after PCI. After analyzing possible external factors, we found that many type 2 bleeding events in the prasugrel group were associated with the PCI technique. The higher incidence of type 2 bleeds in the prasugrel group in PRASFIT-ACS may also have been related to prolonged hemostasis time.

The results of the ROC curve analysis also suggest that type 2 bleeding events were significantly associated with the PRU, especially in the acute stage of antiplatelet therapy (i.e., within 3 days after the LD). In the steady state period of antiplatelet therapy, the incidence of type 2 bleeding events tended to be greater in patients with lower PRU ( $\leq 139)$ than in patients with higher PRU (>139). These findings suggest that medical interventions, such as extending hemostasis time, might be an important factor when treating patients with low platelet 
Citation: Miyazaki S, Isshiki T, Kimura T, Ogawa H, Yokoi H, et al. (2015) Re-evaluation of Bleeding Events in the Japanese PRASFIT-Elective and PRASFIT-ACS Clinical Trials using the Bleeding Academic Research Consortium Criteria. Cardiovasc Pharm Open Access 4: 162. doi:10.4172/2329-6607.1000162

Page 7 of 8

\begin{tabular}{|c|c|c|c|c|c|c|c|c|c|c|}
\hline \multirow{3}{*}{$\begin{array}{l}\text { BARC } \\
\text { category }\end{array}$} & \multicolumn{6}{|c|}{ PRASFIT-ACS } & \multicolumn{4}{|c|}{ PRASFIT-Elective } \\
\hline & \multicolumn{2}{|c|}{ Prasugrel $(n=685)$} & \multicolumn{2}{|c|}{ Clopidogrel $(n=678)$} & \multirow[t]{2}{*}{$\mathrm{HR}(95 \% \mathrm{Cl})$} & \multirow[t]{2}{*}{$P^{*}$} & \multicolumn{2}{|c|}{ Prasugrel $(n=370)$} & \multicolumn{2}{|c|}{ Clopidogrel $(n=372)$} \\
\hline & $\mathrm{N}(\%)$ & $95 \% \mathrm{Cl}$ & $N(\%)$ & $95 \% \mathrm{Cl}$ & & & $N(\%)$ & $95 \% \mathrm{Cl}$ & $N(\%)$ & $95 \% \mathrm{Cl}$ \\
\hline Type $3+5$ & $43(6.3)$ & $4.6-8.4$ & $37(5.5)$ & $3.9-7.4$ & $1.07(0.69-1.67)$ & 0.654 & $10(2.7)$ & $1.3-4.9$ & $12(3.2)$ & $1.7-5.6$ \\
\hline Type 3 & $39(5.7)$ & $4.1-7.7$ & $35(5.2)$ & $3.6-7.1$ & $1.06(0.69-1.67)$ & 0.808 & $10(2.7)$ & $1.3-4.9$ & $12(3.2)$ & $1.7-5.6$ \\
\hline Type 5 & $4(0.6)$ & $0.2-1.5$ & $2(0.3)$ & $0.0-1.1$ & $1.30(0.22-7.80)$ & 0.471 & $0(0.0)$ & $(0.0-1.0)$ & $0(0.0)$ & $0.0-1.0$ \\
\hline Type 4 & $7(1.0)$ & $0.4-2.1$ & $4(0.6)$ & $0.2-1.5$ & $1.76(0.51-6.00)$ & 0.373 & $0(0.0)$ & $(0.0-1.0)$ & $0(0.0)$ & $0.0-1.0$ \\
\hline Type 2 & $73(10.7)$ & $8.4-13.2$ & $44(6.5)$ & $4.8-8.6$ & $1.65(1.14-2.40)$ & 0.013 & $31(8.4)$ & $5.8-11.7$ & $23(6.2)$ & $4.0-9.1$ \\
\hline
\end{tabular}

Bleeding events were recorded from the day of the start of administration of the investigational product to 14 days after the end or discontinuation of administration.

*Generalized Wilcoxon test.

$\mathrm{BARC}=$ Bleeding Academic Research Consortium; $\mathrm{Cl}=$ confidence interval; $\mathrm{HR}=$ hazard ratio

Table 4: Incidence of bleeding events defined using the Bleeding Academic Research Consortium criteria in PRASFIT-ACS and PRASFIT-Elective.

aggregation during the acute phase of PCI for ACS.

There was a higher incidence of bleeding events in PRASFIT-ACS than in PRASFIT-Elective, particularly more severe bleeds (type 3 or 5 ). This is likely to be a result of the inclusion of patients with more severe disease in PRASFIT-ACS and consequently more difficult procedures performed with greater urgency, and necessitating more frequent use of the femoral vascular access route.

Since their development, the BARC criteria have been used to assess bleeding events after PCI in several trials in patients treated with different antithrombotic regimens, and their prognostic value has also been assessed [14-19]. In a study of patients with high platelet reactivity, BARC type 3 or 5 bleeding events were more common in patients treated with high-dose clopidogrel than in those switched to prasugrel [17]. In 444 patients with ACS treated with PCI and prasugrel $10 \mathrm{mg} /$ day, no major bleeding complication (BARC grade 3 or 5) was found after 30 days [18]. One study found that bleeding risk after PCI as defined by the BARC criteria was consistently higher in women than in men, despite matching for age, body mass index, and type of antithrombotic therapy [19]. Trials using the BARC criteria to predict mortality have also been conducted. Both the BARC and TIMI bleeding classification have identified ST-segment elevation myocardial infarction patients at risk of 1-year mortality [14], with BARC type 3b bleeding found to be the strongest predictor in one study [15]. Another study using the BARC criteria found post-PCI bleeding to be an important prognostic factor in patients $>75$ years of age, with the risk for bleeding in that age category higher in women and in patients with impaired renal function [16].

As with all the available criteria for assessing bleeding following PCI, the BARC criteria have some limitations, such as assessing the complications of PCI in ACS patients. It may be necessary to evaluate all possible factors so that spontaneous bleeding can be distinguished in clinical trials involving surgery.

\section{Limitations}

This analysis had several limitations. First, the analyses were done in a post-hoc manner, with bleeding events re-classified after the study keys were opened. However, all bleeding events were assessed in a blinded manner by the Safety Evaluation Committee. Second, the durations of the trials were relatively short, limiting the opportunity to evaluate bleeding events. Finally, comparisons between the prasugrel and clopidogrel groups were not made because clopidogrel was used as a reference drug and was not indicated for patients with stable CAD undergoing PCI in any country at the time this study was planned and started. Despite these limitations, this study provides the first results of the application of the BARC criteria to bleeding events occurring after the same treatment (prasugrel) in Japanese patients with ACS or those undergoing elective procedures.

\section{Conclusion}

Our analysis demonstrated that reclassification of bleeding events using the BARC criteria yielded essentially the same results as those reported using the original TIMI criteria. The incidence of bleeding events classified using both TIMI and BARC criteria was higher in PRASFIT-ACS than in PRASFIT-Elective, as would be expected in a population with more severe disease. The incidences of type 3 or 5 events according to the BARC criteria were similar in the prasugrel and clopidogrel groups. During the acute period of PCI for ACS, medical interventions might be needed to reduce the risk of type 2 bleeding events in patients with low platelet aggregation.

\section{Acknowledgments}

The authors thank Nicholas D. Smith, PhD, and Marion Barnett, PhD, for providing medical writing support. The authors also acknowledge the support of the institutions and investigators involved in the PRASFIT-ACS and PRASFIT-Elective trials. PRASFIT-ACS and PRASFIT-Elective were sponsored by Daiichi Sankyo Co, Ltd (Tokyo, Japan).

The Safety Event Committee comprised Shunichi Miyazaki and Masakatsu Nishikawa.

\section{Disclosure}

Shunichi Miyazaki has received honoraria and clinical research funding from Daiichi Sankyo Co., Ltd. and MSD K.K. Takaaki Isshiki has received honoraria and clinical research funding from Daiichi Sankyo Co., Ltd., Otsuka Pharmaceutical Co. Ltd, and Sanofi K.K., and honoraria from AstraZeneca K.K. Takeshi Kimura has received honoraria and clinical research funding from Daiichi Sankyo Co., Ltd. and Sanofi K.K. Hisao Ogawa has received honoraria from Daiichi Sankyo Co., Ltd. AstraZeneca K.K., Bayer Yakuhin, Ltd., Boehringer Ingelheim Japan, Bristol-Myers Squibb Company, Dainippon Sumitomo Pharma Co., Ltd., Eisai Co., Ltd., Kyowa Hakko Kirin Co., Ltd., Mitsubishi Tanabe Pharma Corporation, MSD K.K., Pfizer Japan Inc., Sanofi K.K., Takeda Pharmaceutical Co., Ltd., and Teijin Pharma Co., Ltd., clinical research funding from Daiichi Sankyo Co., Ltd., and other research funding from Astellas Pharma Inc., AstraZeneca K.K., Boehringer Ingelheim Japan, Bristol-Myers Squibb Company, Chugai Pharmaceutical Co., Ltd., Daiichi Sankyo Co., Ltd., Dainippon Sumitomo Pharma Co., Eisai Co., Ltd., Kowa Company, Ltd. Mitsubishi Tanabe Pharma Corporation, MSD K.K., Novartis Pharma K.K., Otsuka Pharmaceutical Co., Ltd., Pfizer Japan Inc., Sanofi K.K., Shionogi \& Co., Ltd., and Takeda Pharmaceutical Co., Ltd. Hiroyoshi Yokoi has no conflicts of interest to declare. Masakatsu Nishikawa has received honoraria from Daiichi Sankyo Co. Ltd. and Otsuka Pharmaceutical Co., Ltd., and clinical research funding from Otsuka Pharmaceutical Co., Ltd. Masato Nakamura has received honoraria from Daiichi Sankyo Co., Ltd., AstraZeneca K.K., and Sanofi K.K. Yuko Tanaka is an employee of Daiichi Sankyo Co., Ltd. Shigeru Saito has received honoraria from Abbot Vascular Japan, Boston Scientific Japan, and Medtronic.

\section{References}

1. Eikelboom JW, Mehta SR, Anand SS, Xie C, Fox KA, et al. (2006) Adverse impact of bleeding on prognosis in patients with acute coronary syndromes. Circulation 114: 774-782.

2. Rao SV, O'Grady K, Pieper KS, Granger CB, Newby LK, et al. (2005) Impact of bleeding severity on clinical outcomes among patients with acute coronary syndromes. Am J Cardiol 96: 1200-1206.

3. Kwok CS, Rao SV, Myint PK, Keavney B, Nolan J, et al. (2014) Major bleeding after percutaneous coronary intervention and risk of subsequent mortality: a 
Citation: Miyazaki S, Isshiki T, Kimura T, Ogawa H, Yokoi H, et al. (2015) Re-evaluation of Bleeding Events in the Japanese PRASFIT-Elective and PRASFIT-ACS Clinical Trials using the Bleeding Academic Research Consortium Criteria. Cardiovasc Pharm Open Access 4: 162. doi:10.4172/2329-6607.1000162

Page 8 of 8

systematic review and meta-analysis. Open Heart 1: e000021.

4. Anderson JL, Wright RS, Adams CD, Adams CD, Bridges CR, et al. (2012) 2012 ACCF/AHA focused update of the guideline for the management of patients with unstable angina/non-ST-elevation myocardial infarction (updating the 2007 guideline and replacing the 2011 focused update): A report of the American College of Cardiology Foundation/American Heart Association Task Force on Practice Guidelines. J Am Coll Cardiol 60: 645-681.

5. Kusher FG, Hand M, Smith SC Jr, King SB, Anderson JL, et al. (2009) 2009 Focused updates: ACC/AHA Guidelines for the Management of Patients With ST-Elevation Myocardial Infarction (updating the 2004 Guideline and 2007 Focused Update) and ACC/AHA/SCAI Guidelines on Percutaneous Coronary Intervention (updating the 2005 Guideline and 2007 Focused Update): A report of the American College of Cardiology Foundation/American Heart Association Task Force on Practice Guidelines. Circulation 120: 2271-2306.

6. Park KW, Kim HS (2012) Options to overcome clopidogrel response variability. Circ J 76: 287-292.

7. Wiviott SD, Braunwald E, McCabe CH, Montalescot G, Ruzyllo W, et al. (2007) Prasugrel versus clopidogrel in patients with acute coronary syndromes. N Engl J Med 357: 2001-2015.

8. Saito S, Isshiki T, Kimura T, Ogawa H, Yokoi H, et al. (2014) Efficacy and safety of adjusted-dose prasugrel compared with clopidogrel in Japanese patients with acute coronary syndrome: the PRASFIT-ACS study. Circ J 78: 1684-1692.

9. Isshiki T, Kimura K, Ogawa H, Yokoi H, Nanto S, et al. (2014) Prasugrel, a thirdgeneration $\mathrm{P} 2 \mathrm{Y} 12$ receptor antagonist, in patients with coronary artery disease undergoing elective percutaneous coronary intervention phase III, randomized, double-blind study. Circ J 78: 2926-2934.

10. Bovill EG, Terrin ML, Stump DC, Berke AD, Frederick M, et al. (1991) Hemorrhagic events during therapy with recombinant tissue-type plasminogen activator, heparin, and aspirin for acute myocardial infarction. Results of the Thrombolysis in Myocardial Infarction (TIMI), Phase II Trial. Ann Intern Med 115: 256-265.

11. Chesebro JH, Knatterud G, Roberts R, Borer J, Cohen LS, et al. (1987) Thrombolysis in Myocardial Infarction (TIMI) Trial, Phase I: A comparison between intravenous tissue plasminogen activator and intravenous streptokinase. Clinical findings through hospital discharge. Circulation 76: $142-$ 154

12. Mehran R, Rao SV, Bhatt DL, Gibson CM, Caixeta A, et al. (2011) Standardized bleeding definitions for cardiovascular clinical trials: a consensus report from the Bleeding Academic Research Consortium. Circulation 123: 2736-2747.

13. Cuisset T, Grosdidier C, Loundou AD, Quilici J, Loosveld M, et al. (2013) Clinical implications of very low on-treatment platelet reactivity in patients treated with thienopyridine: the POBA study (predictor of bleedings with antiplatelet drugs). JACC Cardiovasc Interv 6: 854-863.

14. Kikkert WJ, van Geloven N, van der Laan MH, Vis MM, Baan J Jr, et al. (2014) The prognostic value of bleeding academic research consortium (BARC) defined bleeding complications in ST-segment elevation myocardial infarction: a comparison with the TIMI (Thrombolysis In Myocardial Infarction), GUSTO (Global Utilization of Streptokinase and Tissue Plasminogen Activator for Occluded Coronary Arteries), and ISTH (International Society on Thrombosis and Haemostasis) bleeding classifications. J Am Coll Cardiol 63: 1866-1875.

15. Matic DM, Milasinovic DG, Asanin MR, Mrdovic IB, Marinkovic JM, et al. (2014) Prognostic implications of bleeding measured by Bleeding Academic Research Consortium (BARC) categorisation in patients undergoing primary percutaneous coronary intervention. Heart 100: 146-152.

16. Ndrepepa G, Neumann FJ, Schulz S, Fusaro M, Cassese S, et al. (2014) Incidence and prognostic value of bleeding after percutaneous coronary intervention in patients older than 75 years of age. Catheter Cardiovasc Interv 83: 182-189.

17. Aradi D, Tornyos A, Pintér T, Vorobcsuk A, Kónyi A, et al. (2014) Optimizing $\mathrm{P} 2 \mathrm{Y} 12$ receptor inhibition in patients with acute coronary syndrome on the basis of platelet function testing: impact of prasugrel and high-dose clopidogrel. J Am Coll Cardiol 63: 1061-1070.

18. Cayla G, Cuisset T, Silvain J, O'Connor SA, Kerneis M, et al. (2013) Prasugre monitoring and bleeding in real world patients. Am J Cardiol 111: 38-44.

19. Ndrepepa G, Schulz S, Neumann FJ, Byrne RA, Hoppmann P, et al. (2013) Bleeding after percutaneous coronary intervention in women and men matched for age, body mass index, and type of antithrombotic therapy. Am Heart J 166 : 534-540. 